Gefässchirurgie $2017 \cdot 22: 316-317$

DOI 10.1007/s00772-017-0302-7

C) Springer Medizin Verlag GmbH 2017

CrossMark

\section{A. Larena-Avellaneda}

Klinik und Poliklinik für Gefäßmedizin - Gefäßchirurgie - Endovaskuläre Therapie - Angiologie, Universitäres Herzzentrum Hamburg, Universitätsklinikum Hamburg-Eppendorf, Hamburg, Deutschland

\title{
Tempora mutantur - Die Zeiten ändern sich
}

Liebe Leserinnen und Leser,

Sie kennen sicher alle diesen Spruch: „Arbeite, um zu leben. Lebe nicht, um zu arbeiten“. Für mich gilt er nicht. Jeden Tag gehe ich gerne zur Arbeit, und ich bin sicher, es geht vielen von Ihnen ähnlich - und wir sehen im Beruf des (Gefäß-)Chirurgen unsere Erfüllung und leben, um zu arbeiten. Wie sagten meine alten Oberärzte so gern, wenn jemand mit neuen Ideen kam - „Das war schon immer so". Aber die Zeiten ändern sich. Das lateinische Sprichwort lautet ja weiter: "nos et mutamur in illis“ - und wir ändern uns in ihnen.

Diese Ausgabe ist „dem Nachwuchs verpflichtet". Wie haben die Zeiten die Umstände unseres Berufes und insbesondere das Selbstverständnis junger Ärztinnen und Ärzte geändert? Während der Ablauf des Studiums im Laufe der Jahrzehnte nur wenig umgestaltet wurde, kann man die Arbeit als Arzt heute sicher nicht mehr mit den 90er-Jahren vergleichen. Dies bezieht auf verschiedene Aspekte. Zum einen haben sich die äußeren Einflüsse erheblich gewandelt. Stand früher die Behandlung am Patienten (auch zeitlich) im Vordergrund, haben sich die Aufgaben des Arztes durch Arbeitsverdichtung, Gewinnoptimierung, Dokumentationsaufwand, rechtliche Aspekte, frühe Spezialisierung usw. deutlich geändert. Heutzutage ist man nicht nur Arzt, sondern auch Sekretär/In, Manager, Buchhalter, Jurist, Blitzableiter und Verwalter. Viel Zeit für die Kernkompetenzen eines Chirurgen bleibt dann im Klinikalltag nicht.

Aber auch die „Human Resources“ haben sich geändert. Inzwischen hat die Generation Y Einzug ins Berufsleben gehal- ten. Während es sicher noch viele junge Kolleginnen und Kollegen gibt, die ihre Erfüllung im Beruf sehen, ist mit der neuen Generation dennoch ein Wertewandel eingetreten. War es früher für einen jungen Arzt das Größte, möglichst lange in der Klinik zu bleiben, um insbesondere viel zu operieren (und die Überstunden wurden weder bezahlt, noch ausgeglichen), ist für viele heute der Wunsch nach einer besseren Work-Life-Balance sehr wichtig. Ein Leben neben der Klinik muss möglich sein und wird auch konsequent eingefordert und angestrebt („Arbeite, um zu leben“). Bereitschaftsdienste mit 36-Stunden-Schichten sind Gottsei-Dank passé. Das Arbeitszeitschutzgesetz schreibt die Arbeitszeiten genau vor. Die jungen Ärztinnen/der junge Arzt von heute verfügen somit meist über mehr Freizeit als dies noch vor relativ kurzer Zeit der Fall war. Hinzu kommt ein inzwischen doch deutlich verbessertes Verdienst in diesem Alter, von dem wir damals als „Ärzte im Praktikum“ nur hätten träumen können. Für die eben genannte Work-Life-Balance ist dies die richtige Voraussetzung.

Dennoch impliziert das Mehr an Freizeit ein Minus an anderen Dingen - sowohl für den Nachwuchs selbst, als auch für die Patienten. Wir alle wissen, dass die beste Einschätzung eines Patienten nur durch wiederholte, regelmäßige Untersuchungen gelingt. Dies ist in den heutigen Zeiten nahezu unmöglich geworden. Informationsverluste durch mehrere Übergaben und die Verlagerung der Arbeit am Patienten zur Arbeit am PC sind die Folge. Bisweilen kennen die Patienten die Namen ihrer Stationsärzte nicht mehr - durch die ständigen Wechsel verliert man den Überblick. Die Oberarzt- und
Chefvisiten werden vom Blatt abgelesen, weil man die Patienten nicht mehr so gut kennt. Wir haben also viel mehr Ärzte, die ihre Patienten aber nicht mehr so gut kennen. Dies ist so gewollt und akzeptiert. Gewollt ist auch die frühe spezialisierte Ausbildung laut Weiterbildungsordnung: Berufsanfänger in der Gefäßchirurgie haben nie gelernt, einen Bauch zu beurteilen - einer der Kernpunkte in der Weiterbildung zum Allgemeinchirurgen, die heute der Gefäßchirurgie nicht mehr vorgeschaltet ist.

Ein weiterer wichtiger Aspekt dieser frühen Spezialisierung ergibt sich für die Akquise des Nachwuchses: Bereits nach dem PJ sollen/müssen sich junge Ärztinnen/Ärzte entscheiden, welche Fachrichtung sie einschlagen möchten. Der Blick in andere chirurgische Gebiete, so wie wir es noch kennengelernt haben VOR der Entscheidung für die Gefäßchirurgie, ist in vielen Kliniken kaum noch möglich. Das heißt, wir müssen den Nachwuchs im Grunde noch VOR dem letzten Staatsexamen für unser Fachgebiet gewinnen. Von daher ist eine Präsenz unseres Faches in den jeweiligen Curriculae der Universitäten von großer Wichtigkeit und muss unsere Bemühungen, die Gefäßchirurgie als Fach an den Universitäten als Lehrstühle zu verankern, bestärken. Auch hier sind wir dem Nachwuchs, wenn auch noch früher, verpflichtet.

Durch die heutigen Abläufe werden, wie oben bereits erwähnt, innerhalb einer Klinik mehr Ärzte eingestellt. An unserer Universitätsklinik haben wir auch einen klaren Weiterbildungsauftrag. Bei gleichem operativem Aufkommen heißt das natürlich, dass pro Arzt in der Weiterbildung weniger Operationen bzw. Interventionen anfallen. Damit wird es oft 
schwierig, die Anzahl der Eingriffe innerhalb der minimalen Weiterbildungszeit durchzuführen. Hier sind dann neue Konzepte, dem Nachwuchs verpflichtet, extrem wichtig. Nur durch eine strukturierte Ausbildung und Zuteilung der Eingriffe kann eine adäquate Ausbildung erfolgen. Viele der offenen Aorteneingriffe, die früher nur von Oberärzten operiert wurden, werden heute Jüngeren assistiert. Dies erinnert wenig an früher, als man es schaffen musste, 5 Magenresektionen bzw. 5 Mamma-Operationen für den Allgemeinchirurgen durchgeführt zu haben. Auf der anderen Seite müssen wir den Nachwuchs aber auch so gut ausbilden, dass er gegenüber anderen, vor allem endovaskulär tätigen Fächern, wettbewerbsfähig ist. Dem trägt die neue Weiterbildungsordnung Rechnung, in der der Stellenwert der Interventionen deutlich angehoben wurde. Ein weiterer Weg, den Nachwuchs wieder auf die Kernkompetenzen zu fokussieren, besteht in der Delegation von bestimmten Tätigkeiten. Dieser Weg wurde durch die Einführung der Ausbildung von Pflegekräften zum Gefäßassistent/in DGG ${ }^{\circledR}$ oder auch endovaskulären Assistenten (EVA) von unserer Fachgesellschaft eingeschlagen. Zusammen auch mit dem „Physician assistant“, dem zuletzt sogar die Titelseite und zwei Artikel im „Deutschen Ärzteblatt“ gewidmet waren [1], können so viele Arbeiten abgegeben werden. In der Folge wären für die Aufrechterhaltung des Routineablaufes weniger Ärzte notwendig, die dann wiederum vermehrt Patienten betreuen und operieren können. Allerdings ist Vorsicht geboten - es ist für die Arbeitgeber natürlich sehr verführerisch, auf diese Weise Ärzte durch preiswerte Arbeitskräfte zu ersetzen. Von daher müssen die Tätigkeitsfelder eindeutig definiert werden.

Es ist also unsere Aufgabe, vor dem Hintergrund der oben genannten Herausforderungen unser Fach attraktiv zu gestalten: Die Begeisterung für die Gefäßchirurgie bei den Studentinnen und Studenten frühzeitig zu wecken, den jungen Ärztinnen und Ärzten eine gute, zeitgerechte Ausbildung zu ermöglichen, und an den großen Kliniken und Universitäten Forschung und Lehre konsequent und nachhaltig zu ermöglichen. $\mathrm{Ob}$ die jungen Kolleginnen und Kollegen heute mit mehr Geld und mehr Freizeit glücklicher sind, als die jungen Ärzte früher unter anderen Arbeitsbedingungen (aber mehr klinischen Aufgaben), sei dahingestellt - „Tempora mutantur“.

Von daher freue mich sehr über das wichtige Motto unseres diesjährigen Jahreskongresses und die Artikel in der vorliegenden Ausgabe, die sich ganz dem Thema widmen - Unser Präsident, Prof. Schmitz-Rixen, wird Sie auf den folgenden Seiten zum Thema hinführen. Mit Latein habe ich begonnen und möchte mit einem englischen Zitat eines sehr bekannten Zeitgenossen (Frank Veith) enden, das explizit für unseren Nachwuchs gilt: „Become endocompetent, or become extinct".

\section{Herzlichst,}

Ihr

Axel Wurra-Mvelenude

Prof. Dr. A. Larena-Avellaneda

\section{Korrespondenzadresse}

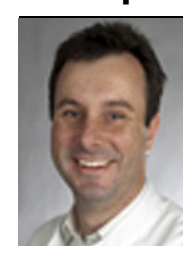

Prof. Dr. A. Larena-

Avellaneda

Klinik und Poliklinik

für Gefäßmedizin

- Gefäßchirurgie -

Endovaskuläre Therapie -

Angiologie, Universitäres

Herzzentrum Hamburg,

Universitätsklinikum

Hamburg-Eppendorf

Martinistraße 52,

20246 Hamburg, Deutschland larena@uke.de

Interessenkonflikt. A. Larena-Avellaneda gibt an dass kein Interessenkonflikt besteht.

\section{Literatur}

1. Korzilius H, Osterloh F (2017) Physician Assistant: Ärzte müssen von Tätigkeiten entlastet werden, die nicht zu ihren Kernaufgaben gehören. Dtsch Arztebl 114(26):1082-1085
Leukämie: RNA-Atlas stützt Therapieentscheidungen

Einen umfassenden Atlas von Ribonukleinsäuren (RNA) haben Forscher der Medizinischen Hochschule Hannover erstellt. Mit diesem sei die Entstehung von Blutzellen und Blutkrankheiten besser nachvollziehbar.

Die MHH-Forscher konnten mit ihren Analysen vielen nichtkodierenden Ribonukleinsäuren (RNAs) Funktionen zuordnen. Beispielsweise fanden sie nichtkodierende RNAs, die für die Ausreifung der Granulozyten wichtig sind. Insgesamt gibt es rund 40.000 nichtkodierende RNAs. Sie steuern viele sehr unterschiedliche Vorgänge in den Zellen - beispielsweise, welche Gene wann wie oft abgelesen werden. „Mit unserem Atlas kann man besser verstehen, wie Blutzellen, aber auch Blutkrankheiten entstehen. Es ist auch möglich, die Verläufe von Krankheiten bei Patienten mit Blutkrebs besser vorherzusagen und so individueller zu therapieren, sagt Privatdozent Dr. Jan-Henning Klusmann von der MHH-Klinik für Pädiatrische Hämatologie und Onkologie. Die Ausreifung von Blutzellen im Knochenmark basiert auf einem komplexen und bisher wenig verstandenen Zusammenspiel von nichtkodierenden RNAs und anderen Molekülen in der Zelle. „Unser Ziel ist es, mit Hilfe des Atlas die Ursache von Blutkrebs bei einzelnen Patienten festzustellen und daraus Empfehlungen für die Therapie abzuleiten", so Klusmann.

Der Atlas ist im Internet einzusehen unter: www.leukemia-research.de (Stichwort: "IncScape").

Quelle: Medizinische Hochschule Hannover (www.mh-h.de)

basierend auf: Nature Communications (2017) 8:218, doi 10.1038/s41467-017-00212-4 